\title{
PRODUCTION PLANNING WITH REMANUFACTURING UNDER UNCERTAIN DEMAND AND RETURNS*
}

\author{
Oğuz SOLYALI ${ }^{\dagger}$
}

\begin{abstract}
In this study, a production planning problem in which a producer remanufactures returned products into serviceable products besides manufacturing serviceable products is considered. The main challenge in this planning problem is the uncertain demand for the serviceable products and the uncertain returns of the used products. The probability distributions of the uncertain demand and uncertain returns are not known. Only the means of uncertain parameters and maximum deviations from these means are known or can be estimated. The problem is to determine the quantity of serviceable products that are manufactured, the quantity of returned products that are remanufactured, and the quantity of returned products that are disposed over a multi-period planning horizon such that total cost composed of production, inventory and disposal costs is minimized. A new robust linear programming model that yields a feasible production-disposal policy regardless of the realization of demand and returns is proposed and compared with a robust linear programming model existing in the literature. The computational results reveal that the proposed model significantly outperforms the one existing in literature in terms of the actual cost savings.
\end{abstract}

Keywords: Production planning, uncertain demand and returns, robust optimization, linear programming.

$\ddot{O} z$

Belirsiz Talep ve Geri Dönen Ürünler Durumunda Yeniden Üretim İle Üretim Planlaması

\footnotetext{
* This work was supported by the Campus Research Fund of Middle East Technical University, Northern Cyprus Campus under Grant BAP-SOSY-12-YG-3. This support is gratefully acknowledged.

${ }^{\dagger}$ Assoc.Prof.Dr., Middle East Technical University, Northern Cyprus Campus, Business Administration Program, Kalkanl1, KKTC 99738, solyali@metu.edu.tr
} 
$\mathrm{Bu}$ çalışmada, bir üreticinin satılabilir ürünleri üretmesi yanında, geri dönen ürünleri yeniden üreterek satılabilir ürünlere dönüştürdüğü bir üretim planlama problemi ele alınmıştır. Bu planlama problemindeki en büyük zorluk, satılabilir ürünler için talebin belirsiz olması ve geri dönen ürün miktarlarındaki belirsizliktir. Belirsiz talep ve geri dönen ürün miktarlarındaki belirsizliğin olasılıksal dağılımları bilinmemektedir. Bu belirsiz parametrelerin sadece ortalamaları ve bu ortalamalardan azami sapmaları bilinmekte veya tahmin edilebilmektedir. Problem, toplam üretim, envanter ve imha maliyetlerini enazlayacak şekilde, planlama ufku boyunca, üretilecek satılabilir ürünlerin miktarının, yeniden üretilecek geri dönen ürünlerin miktarının ve imha edilecek geri dönen ürünlerin miktarının belirlenmesidir. Talep ve geri dönen ürün miktarlarının gerçekleşmelerinden bağımsız olarak, olurlu bir üretim-imha politikası veren, yeni bir gürbüz doğrusal programlama modeli önerilmekte ve literatürde varolan bir gürbüz doğrusal programlama modeli ile karşılaştırılmaktadır. Sayısal sonuçlar, önerilen modelin, literatürde varolan modelden gerçek maliyet tasarrufu olarak önemli derecede üstün olduğunu göstermektedir.

Anahtar Sözcükler: Üretim planlama, belirsiz talep ve geri dönen ürünler, gürbüz eniyileme, doğrusal programlama.

\section{INTRODUCTION}

Remanufacturing, which can be defined as the activity of transforming returned products into serviceable (i.e., sellable) products, has recently become popular in several manufacturing industries due to concerns on sustainability, environmental protection, as well as cost saving opportunities in production. A number of successful companies from different industries (e.g., Dell, General Motors, Kodak, Xerox) adopted remanufacturing as a part of their usual production planning activity (Akçalı, Çetinkaya, 2011). For example, BMW considered reuse of end-of-life cars, Kodak remanufactured one-off cameras, and Xerox recovered toner cartridges, besides their regular manufacturing activities (Wei et al., 2011).

There is certainly a need for producers to jointly consider their manufacturing and remanufacturing activities in order to take full advantage of cost savings in production and to protect the environment by lowering the consumption of natural resources. Although there are many studies jointly considering manufacturing and remanufacturing activities, these studies assume either a fully deterministic setting (e.g., Helmrich et al., 2014; Li et al., 2014) in which the demand for the serviceable products and the quantities of returned products are known with certainty or a stochastic setting (e.g., Naeem et al., 2013; Shi et al., 2011) in which the probability distributions of the demand for 
the serviceable products and the quantities of returned products are known. For a recent review on the integrated manufacturing and remanufacturing planning with both deterministic and stochastic environments, refer to Akçalı and Çetinkaya (2011). In real-life, demand and product returns are uncertain, and probability distributions of these two uncertain parameters are mostly not available (Wei et al., 2011). Thus, there is a need to consider uncertain demand and product returns while deciding on the quantities to manufacture, the quantities to remanufacture from returned products and the quantities of returned products to dispose in each period (i.e., day, week or month). In this study, the production planning problem with remanufacturing under uncertain demand and returns is addressed using state-of-the-art linear programming (LP) models and robust optimization methodologies.

Robust optimization (RO) is a powerful tool for optimization problems with uncertain parameters where probability distributions of uncertain parameters are unknown. RO attempts to find the best solution that is (most likely) feasible regardless of the realization of uncertain parameters within their given uncertainty sets. Soyster (1973), the first study on RO, proposed a conservative approach by taking the worst possible values of uncertain parameters. Ben-Tal and Nemirovski $(1998,1999,2000)$ considered RO models for the general convex optimization problems under ellipsoidal uncertainty set. Bertsimas and Sim (2004) developed the budget of uncertainty RO approach for the linear optimization problems under interval uncertainty set. In this approach a limited number of the uncertain parameters, defined by the budget parameter, are allowed to deviate from their mean values simultaneously, and it is possible to ensure a high probability of feasibility for a constraint by selecting an appropriate budget. Bertsimas and Sim (2003) extended the budget of uncertainty RO approach to discrete optimization and network flow problems with uncertain objective function coefficients. Taking into account that the real values of uncertain parameters are revealed sequentially over time and some decisions can be postponed until observing these real values, Ben-Tal et al. (2004) proposed the adjustable robust counterpart (ARC) for multistage linear programs with uncertain parameters. In the ARC some variables, called adjustable variables, can be determined after observing the real value of some uncertain parameters whereas the rest of the variables, called nonadjustable variables, must be determined at the beginning. Because the ARC is mostly intractable, Ben-Tal et al. (2004), proposed a tractable approximation of the ARC, called the affinely adjustable robust counterpart (AARC). In AARC, adjustable variables are redefined as affine functions of realized parameters. Bertsimas et al. (2010), showed that the AARC yields the optimal solution under certain circumstances. 
Robust inventory management and production planning problems under demand uncertainty, have been addressed by many researchers using the RO approaches (e.g., Ben-Tal et al., 2004; 2005; Bertsimas, Thiele 2006; Bienstock, Özbay, 2008; Ben-Tal et al., 2009; See, Sim 2010). While there are numerous studies considering integrated manufacturing and remanufacturing planning under known demand and returns or under uncertain demand and returns with known probability distributions, to the best of our knowledge, the paper by Wei et al. (2011) is the only study considering this production planning problem under uncertain demand and returns with known supports (i.e., only means and maximum deviations from means are known). Wei et al. (2011) addressed this problem using the budget of uncertainty RO methodology. The main deficiency of the work by Wei et al. (2011) is that their model yields a static solution in that they make all the decisions at the beginning. However, the problem is a multi-period planning problem and uncertainty regarding demand and returns are realized sequentially (i.e., demand and returns in the first period are known after a period, demand and returns in the first two periods are known after the second period, and so on) as the time passes, and this information could be utilized for better planning.

In this paper, we consider the same production planning problem as Wei et al. (2011). The problem is to determine the quantity of serviceable products that are manufactured, the quantity of returned products that are remanufactured into serviceable products, and the quantity of returned products that are disposed over a multi-period planning horizon such that total cost composed of production, inventory and disposal costs is minimized. We present the robust LP model of Wei et al. (2011), show that their model has some flaws and correct their model. We propose a new robust LP model that makes use of the multi-stage nature of the problem. Specifically, we propose an affinely adjustable robust model that yields a production-disposal policy by defining manufacturing, remanufacturing and disposal variables in terms of realized demand and return values. We compare the corrected model of Wei et al., (2011) and our model on a computational study which reveals that our model yields significantly superior practical results than that of Wei et al. (2011).

The rest of the paper is organized as follows. We give the problem description and formulation in Section 1. Section 2 presents the existing robust model, its derivation and the flaw it has. We propose a new robust model in Section 3. Section 4 presents computational results on test instances obtained by the existing and newly proposed models. Section 5 concludes the paper. Finally, we present the detailed derivation of the new robust model in Appendix.

\section{PROBLEM DESCRIPTION AND FORMULATION}


In the production planning problem considered in this paper, a producer faces dynamic stochastic demand for a serviceable product and dynamic stochastic returns of the used product in each period over a finite time horizon. The probability distributions of demand and returns are not known. Only the mean values and the maximum deviations from the mean values are known or can be estimated for demand and returns in each period. The producer can manufacture serviceable products and/or remanufacture serviceable products from the returned products in order to satisfy the demand it faces. There is no quality difference between the manufactured serviceable products and the remanufactured ones. When the producer (re)manufactures, a variable (re)manufacturing cost per unit (re)manufactured is incurred. The producer must decide on the quantity to manufacture and remanufacture at the beginning of each period $t$. Once the demand in period $t$ has been observed, the inventory level of the serviceable product at the end of period $t$, which is equal to inventory level of the serviceable product at the end of period $t-1$ plus manufacturing and remanufacturing quantities in period $t$ less realized demand in period $t$, is obtained. If the ending inventory level is positive (resp. negative), an inventory holding (resp. backlogging) cost per unit is incurred. The producer can remanufacture or dispose the returned products and must decide on the quantity to remanufacture and dispose at the beginning of each period $t$. A variable disposal cost per unit disposed is incurred when the producer disposes the returned products. Once the returns in period $t$ has been observed, the inventory level of the returned products at the end of period $t$, which is equal to inventory level of the returned products at the end of period $t-1$ plus realized return in period $t$ less remanufacturing and disposal quantities in period $t$, is obtained. If the ending inventory level of returned products is positive, an inventory holding cost per unit is incurred. Note that the ending inventory level of returned products cannot be negative. Without loss of generality, we assume that manufacturing, remanufacturing and disposal of products occur instantaneously. In the following, important parameters and decision variables that are used in the model are given.

\section{Parameters:}

$h^{R}$ : Unit inventory holding cost of returned products.

$h^{S}$ : Unit inventory holding cost of serviceable products.

$b$ : Unit backlogging cost of serviceable products.

$c^{R}$ : Unit cost of remanufacturing a returned product.

$c^{M}$ : Unit cost of manufacturing a serviceable product.

$c^{D}$ : Unit cost of disposing a returned product.

$I_{0}^{R}$ : Inventory level of returned products at the beginning of the planning horizon. 
$I_{0}^{S}$ : Inventory level of serviceable products at the beginning of the planning horizon.

$d_{t}$ : Uncertain demand for the serviceable products in period $t$.

$\bar{d}_{t}: \quad$ Estimate of the mean demand in period $t$.

$\hat{d}_{t}$ : Maximum deviation from the mean demand in period $t$.

$r_{t}:$ Uncertain quantity of returned products in period $t$.

$\bar{r}_{t}$ : Estimate of the mean quantity of returned products in period $t$.

$\hat{r}_{t}:$ Maximum deviation from the mean quantity of returned products in period $t$.

\section{Decision Variables:}

$X_{t}^{M}$ : Quantity of serviceable products that are manufactured in period $t$.

$X_{t}^{R}$ : Quantity of returned products that are remanufactured in period $t$.

$X_{t}^{D}$ : Quantity of returned products that are disposed in period $t$.

$I_{t}^{S}$ : Inventory holding or backlogging cost of serviceable products at the end of period $t$.

The problem can be formulated as follows:

$$
\begin{array}{ll}
\operatorname{Min} \sum_{t=1}^{T}\left[h^{R}\left(I_{0}^{R}+\sum_{i=1}^{t}\left(r_{i}-X_{i}^{R}-X_{i}^{D}\right)\right)+I_{t}^{S}+c^{M} X_{t}^{M}+c^{R} X_{t}^{R}+c^{D} X_{t}^{D}\right] \\
\text { s.t. } \\
I_{t}^{S} \geq h^{S}\left[I_{0}^{S}+\sum_{i=1}^{t}\left(X_{i}^{M}+X_{i}^{R}-d_{i}\right)\right] & 1 \leq t \leq T, d \in D_{t}, \\
I_{t}^{S} \geq-b\left[I_{0}^{S}+\sum_{i=1}^{t}\left(X_{i}^{M}+X_{i}^{R}-d_{i}\right)\right] & 1 \leq t \leq T, d \in D_{t}, \\
I_{0}^{R}+\sum_{i=1}^{t}\left(r_{i}-X_{i}^{R}-X_{i}^{D}\right) \geq 0 & 1 \leq t \leq T, r \in R_{t}, \\
X_{t}^{M} \geq 0, X_{t}^{R} \geq 0, X_{t}^{D} \geq 0 & 1 \leq t \leq T,
\end{array}
$$

where

$$
D_{t}=\left\{\bar{d}_{i}+\hat{d}_{i} z_{i}:\left|z_{i}\right| \leq 1 \forall 1 \leq i \leq t\right\} \text { and } R_{t}=\left\{\bar{r}_{i}+\hat{r}_{i} z_{i}:\left|z_{i}\right| \leq 1 \forall 1 \leq i \leq t\right\} .
$$

The objective function (1) is the total of inventory, production and disposal costs. Constraints (2) and (3) keep track of the inventory cost associated with inventory carried at the end of period $t$ or inventory backordered at the end of period $t$ for any possible realization of demand. Note that $I_{0}^{S}+$ $\sum_{i=1}^{t}\left(X_{i}^{M}+X_{i}^{R}-d_{i}\right)$ is the inventory level of serviceable products at the end of period $t$. Constraints (4) ensure that the inventory level of the returned products is nonnegative at the end of period $t$. Constraints (5) are for nonnegativity of 
Production Planning with Remanufacturing under Uncertain Demand and Returns 281

variables. Note that the model (1)-(5) is intractable because constraints (2)-(4) must hold for infinitely many possible values of uncertain demand and returns.

\section{THE BUDGET OF UNCERTAINTY ROBUST MODEL}

Using the budget of uncertainty RO methodology of Bertsimas and Sim (2003), Wei et al. (2011) reformulated the uncertain model (1)-(5) into a tractable robust LP model. The robust LP model of Wei et al. (2011) makes all production-disposal decisions at time 0 and it ensures the feasibility of these decisions with a high probability for all possible realizations of uncertain demand and returns. To overcome overconservatiness while protecting against infeasibility, Wei et al. (2011) defined $\Gamma_{t}^{d}$ and $\Gamma_{t}^{r}$, respectively, as the maximum number of uncertain demand and uncertain return values that can simultaneously deviate from their mean values (i.e., $\bar{d}_{t}$ and $\bar{r}_{t}$ ) until the end of period $t$. We present the LP model derived by Wei et al., (2011), referred to as BURM, in the following:

$$
\begin{aligned}
& \text { (BURM) Min } \sum_{t=1}^{T}\left[h^{R} \phi_{t T}+I_{t}^{S}+c^{M} X_{t}^{M}+\tilde{c}^{R} X_{t}^{R}+\tilde{c}^{D} X_{t}^{D}\right]+h^{R} \beta_{T} \Gamma_{T}^{r}+T h^{R} I_{0}^{R} \\
& +\sum_{t=1}^{T} h^{R}(T-t+1) \bar{r}_{t} \\
& \text { s.t. (5), } \\
& I_{t}^{S} \geq h^{S}\left[I_{0}^{S}+\sum_{i=1}^{t}\left(X_{i}^{M}+X_{i}^{R}-\bar{d}_{i}\right)+q_{t} \Gamma_{t}^{d}+\sum_{i=1}^{t} \xi_{i t}\right] \quad 1 \leq t \leq T \\
& I_{t}^{S} \geq b\left[-I_{0}^{S}-\sum_{i=1}^{t}\left(X_{i}^{M}+X_{i}^{R}-\bar{d}_{i}\right)+q_{t} \Gamma_{t}^{d}+\sum_{i=1}^{t} \xi_{i t}\right] \quad 1 \leq t \leq T \\
& I_{0}^{R}+\sum_{i=1}^{t}\left(\bar{r}_{i}-X_{i}^{R}-X_{i}^{D}\right) \geq w_{t} \Gamma_{t}^{r}+\sum_{i=1}^{t} \varepsilon_{i t}+X_{t+1}^{R} \\
& q_{t}+\xi_{i t} \geq \hat{d}_{i} \\
& w_{t}+\varepsilon_{i t} \geq \hat{r}_{i} \\
& \beta_{T}+\phi_{t T} \leq(T-t \leq t \leq t \leq T \\
& q_{t} \geq 0, \xi_{i t} \geq 0, w_{t} \geq 0, \varepsilon_{i t} \geq 0, \beta_{T} \geq 0, \phi_{t T} \geq 0
\end{aligned}
$$

where $\tilde{c}^{R}=c^{R}-(T-t+1) h^{R}$, and $\tilde{c}^{D}=c^{D}-(T-t+1) h^{R}$.

Note that the additional variables $q_{t}, \xi_{i t}, w_{t}, \varepsilon_{i t}, \beta_{T}$, and $\phi_{t T}$ for $1 \leq i \leq$ $t \leq T$, and the additional constraints (10)-(12) are used when transforming the uncertain model (1) - (5) into a tractable model. Next we show that there are flaws in constraints (9) and (12) of the model given above. As shown by 
Bertsimas and Sim (2004), it is possible to set $\Gamma_{t}^{r}$ to a value in advance such that the probability of violating the feasibility of (4) is very low. Constraints (9) and (11) are derived from constraints (4) by ensuring the feasibility of (4) for all possible realizations of uncertain returns $r_{i}(1 \leq i \leq t)$ within their interval $\left[\bar{r}_{i}-\hat{r}_{i}, \bar{r}_{i}+\hat{r}_{i}\right]$ provided that at most $\Gamma_{t}^{r}$ uncertain return values can deviate from their mean values until the end of period $t$. Thus, we need to solve

$t \leq T$,

$$
\operatorname{Min}\left\{I_{0}^{R}+\sum_{i=1}^{t}\left(\bar{r}_{i}+\hat{r}_{i} z_{i}-X_{i}^{R}-X_{i}^{D}\right):\left|z_{i}\right| \leq 1, \sum_{i=1}^{t}\left|z_{i}\right| \leq \Gamma_{t}^{r}, 1 \leq i \leq t\right\} \quad 1 \leq
$$

which is equivalent to solving the following auxiliary LP model for each period $t(1 \leq t \leq T)$ :

$$
\begin{aligned}
& -\operatorname{Max} \sum_{i=1}^{t} \hat{r}_{i} z_{i} \\
& \text { s.t. } \sum_{i=1}^{t} z_{i} \leq \Gamma_{t}^{r}, \\
& 0 \leq z_{i} \leq 1 \quad 1 \leq i \leq t .
\end{aligned}
$$

Defining $w_{t}$ and $\varepsilon_{i t}$ as the dual variables associated with constraints (16) and (17), respectively, and using duality theory, we obtain the following dual model:

$$
\begin{array}{ll}
-\operatorname{Min} w_{t} \Gamma_{t}^{r}+\sum_{i=1}^{t} \varepsilon_{i t} & \\
\text { s.t. } w_{t}+\varepsilon_{i t} \geq \hat{r}_{i} & 1 \leq i \leq t, \\
w_{t} \geq 0, \varepsilon_{i t} \geq 0 & 1 \leq i \leq t .
\end{array}
$$

As a result, the first set of constraints of the above dual model is (11) and we obtain $I_{0}^{R}+\sum_{i=1}^{t}\left(\bar{r}_{i}-X_{i}^{R}-X_{i}^{D}\right)-\left(w_{t} \Gamma_{t}^{r}+\sum_{i=1}^{t} \varepsilon_{i t}\right) \geq 0$ in place of (14). Noting that $X_{t+1}^{R}$ term in the right-hand side of (9) should not exist, we can state the following constraints as the correct form of (9):

$$
I_{0}^{R}+\sum_{i=1}^{t}\left(\bar{r}_{i}-X_{i}^{R}-X_{i}^{D}\right) \geq w_{t} \Gamma_{t}^{r}+\sum_{i=1}^{t} \varepsilon_{i t} \quad 1 \leq t \leq T .
$$

Using a similar auxiliary LP model and duality theory, one can easily obtain the correct form of constraints (12) as:

$$
\beta_{T}+\phi_{t T} \geq(T-t+1) \hat{r}_{t} \quad 1 \leq t \leq T .
$$

Note that the only difference between (12) and (12') is the direction of the inequality. It must be a ' $\geq$ ' sign according to the duality theory, as in (12'), 
Production Planning with Remanufacturing under Uncertain Demand and Returns 283

because the dual variables of (12') are restricted to be nonnegative in the auxiliary LP model with a maximization objective (see model (18) in Wei et al. 2011).

It is important to indicate these flaws because we observed the same flaws in another study in the literature. See Chunfa et al. (2011) who proposed a budget of uncertainty robust model to a similar problem with the same flaws in their model.

\section{THE NEW ROBUST MODEL}

We propose a new robust LP model based on defining manufacturing, remanufacturing, disposal and inventory variables as affine functions of realized demand and return values. All variables are adjustable variables, which are decided in each period based on the earlier periods' realized demand and returns. Note that at the beginning (resp. end) of a period $t$, we know demand and returns in periods $1,2 \ldots, t-1$ (resp. $1,2, \ldots, t$ ). Specifically, we propose $X_{t}^{M}, X_{t}^{R}, X_{t}^{D}$ and $I_{t}^{S}$ to be replaced with the following terms to make the model adjustable to the uncertain demand and returns:

$$
\begin{array}{ll}
X_{t}^{M}=X_{t 0}^{M}+\sum_{k=1}^{t-1}\left(d_{k} X_{t k}^{1 M}+r_{k} X_{t k}^{2 M}\right) & 1 \leq t \leq T \\
X_{t}^{R}=X_{t 0}^{R}+\sum_{k=1}^{t-1}\left(d_{k} X_{t k}^{1 R}+r_{k} X_{t k}^{2 R}\right) & 1 \leq t \leq T \\
X_{t}^{D}=X_{t 0}^{D}+\sum_{k=1}^{t-1}\left(d_{k} X_{t k}^{1 D}+r_{k} X_{t k}^{2 D}\right) & 1 \leq t \leq T \\
I_{t}^{S}=I_{t 0}^{S}+\sum_{k=1}^{t}\left(d_{k} I_{t k}^{1 S}+r_{k} I_{t k}^{2 S}\right) & 1 \leq t \leq T,
\end{array}
$$

where $X_{t 0}^{M}, X_{t k}^{1 M}, X_{t k}^{2 M}, X_{t 0}^{R}, X_{t k}^{1 R}, X_{t k}^{2 R}, X_{t 0}^{D}, X_{t k}^{1 D}, X_{t k}^{2 D}, I_{t 0}^{S}, I_{t k}^{1 S}$, and $I_{t k}^{2 S}$ are the decision variables.

Replacing $X_{t}^{M}, X_{t}^{R}, X_{t}^{D}$ and $I_{t}^{S}$ variables with the right-hand sides of (18)(21), respectively, in the model (1)-(5), we obtain an uncertain model which should be reformulated as a tractable model. As shown by Ben-Tal et al. (2004), the following inequality

$$
\alpha_{0}+\sum_{t=1}^{T} \alpha_{t} a_{t} \leq 0
$$

where $a_{t} \in\left[\bar{a}_{t}-\hat{a}_{t}, \bar{a}_{t}+\hat{a}_{t}\right]$ for $1 \leq t \leq T$ is an uncertain parameter and $\alpha_{t}$ for $1 \leq t \leq T$ is an expression involving some decision variables, can equivalently be reformulated as 


$$
\begin{aligned}
& \alpha_{0}+\sum_{t=1}^{T}\left(\alpha_{t} \bar{a}_{t}+\gamma_{t} \hat{a}_{t}\right) \leq 0, \\
& -\gamma_{t} \leq \alpha_{t} \leq \gamma_{t}
\end{aligned}
$$$$
1 \leq t \leq T .
$$

Using the above methodology, we transformed the uncertain model (1)(5) into a tractable new robust LP model, which we refer to as the affinely adjustable robust model (AARM) and present in the following (see Appendix for details on the derivation of this model):

$$
\begin{aligned}
& \text { (AARM)Min } \sum_{t=1}^{T}\left(I_{t 0}^{S}+\sum_{k=1}^{t}\left(\bar{d}_{k} I_{t k}^{1 S}+\bar{r}_{k} I_{t k}^{2 S}\right)+c^{M} \sum_{t=1}^{T}\left(X_{t 0}^{M}+\right.\right. \\
& \sum_{k=1}^{t-1}\left(\bar{d}_{k} X_{t k}^{1 M}+\bar{r}_{k} X_{t k}^{2 M}\right)+\sum_{t=1}^{T} \tilde{c}_{t}^{R}\left(X_{t 0}^{R}+\sum_{k=1}^{t-1}\left(\bar{d}_{k} X_{t k}^{1 R}+\bar{r}_{k} X_{t k}^{2 R}\right)+\right. \\
& \sum_{t=1}^{T} \tilde{c}_{t}^{D}\left(X_{t 0}^{D}+\sum_{k=1}^{t-1}\left(\bar{d}_{k} X_{t k}^{1 D}+\bar{r}_{k} X_{t k}^{2 D}\right)+\sum_{t=1}^{T}\left(\hat{d}_{t} p_{t}^{1}+\hat{r}_{t} p_{t}^{2}\right)+T h^{R} I_{0}^{R}+\right. \\
& \sum_{t=1}^{T} h^{R}(T-t+1) \bar{r}_{t} \\
& \text { s.t. } \\
& -p_{k}^{1} \leq I_{k k}^{1 S}+\sum_{t=k+1}^{T}\left(I_{t k}^{1 S}+c^{M} X_{t k}^{1 M}+\tilde{c}_{t}^{R} X_{t k}^{1 R}+\tilde{c}_{t}^{D} X_{t k}^{1 D}\right) \leq p_{k}^{1} \quad 1 \leq k \leq T,(26) \\
& -p_{k}^{2} \leq I_{k k}^{2 S}+h^{R}(T-k+1)+\sum_{t=k+1}^{T}\left(I_{t k}^{2 S}+c^{M} X_{t k}^{2 M}+\tilde{c}_{t}^{r} X_{t k}^{2 R}+\tilde{c}_{t}^{d} X_{t k}^{2 D}\right) \leq p_{k}^{2} \\
& 1 \leq k \leq T \text {, } \\
& I_{t 0}^{S}+\sum_{k=1}^{t}\left(\bar{d}_{k} I_{t k}^{1 S}+\bar{r}_{k} I_{t k}^{2 S}\right)-h^{S} \sum_{i=1}^{t} \sum_{k=1}^{i-1}\left(\bar{d}_{k} X_{i k}^{1 M}+\bar{r}_{k} X_{i k}^{2 M}+\bar{d}_{k} X_{i k}^{1 R}+\bar{r}_{k} X_{i k}^{2 R}\right) \\
& -h^{S} \sum_{i=1}^{t}\left(X_{i 0}^{M}+X_{i 0}^{R}\right)-\sum_{k=1}^{t}\left(\hat{d}_{k} n_{t k}^{1}+\hat{r}_{k} n_{t k}^{2}\right) \geq h^{S}\left(I_{0}^{S}-\sum_{i=1}^{t} \bar{d}_{i}\right) \quad 1 \leq t \leq T \text {, } \\
& -n_{t k}^{1} \leq h^{S} \sum_{i=k+1}^{t}\left(X_{i k}^{1 M}+X_{i k}^{1 R}\right)-h^{S}-I_{t k}^{1 S} \leq n_{t k}^{1} \quad 1 \leq t \leq T, 1 \leq k \leq t, \\
& -n_{t k}^{2} \leq h^{S} \sum_{i=k+1}^{t}\left(X_{i k}^{2 M}+X_{i k}^{2 R}\right)-I_{t k}^{2 S} \leq n_{t k}^{2} \quad 1 \leq t \leq T, 1 \leq k \leq t, \\
& I_{t 0}^{S}+\sum_{k=1}^{t}\left(\bar{d}_{k} I_{t k}^{1 S}+\bar{r}_{k} I_{t k}^{2 S}\right)+b \sum_{i=1}^{t} \sum_{k=1}^{i-1}\left(\bar{d}_{k} X_{i k}^{1 M}+\bar{r}_{k} X_{i k}^{2 M}+\bar{d}_{k} X_{i k}^{1 R}+\bar{r}_{k} X_{i k}^{2 R}\right) \\
& +b \sum_{i=1}^{t}\left(X_{i 0}^{M}+X_{i 0}^{R}\right)-\sum_{k=1}^{t}\left(\hat{d}_{k} s_{t k}^{1}+\hat{r}_{k} s_{t k}^{2}\right) \geq-b\left(I_{0}^{S}-\sum_{i=1}^{t} \bar{d}_{i}\right) 1 \leq t \leq T, \\
& -s_{t k}^{1} \leq-b \sum_{i=k+1}^{t}\left(X_{i k}^{1 M}+X_{i k}^{1 R}\right)+b-I_{t k}^{1 S} \leq s_{t k}^{1} \quad 1 \leq t \leq T, 1 \leq k \leq t, \\
& -s_{t k}^{2} \leq-b \sum_{i=k+1}^{t}\left(X_{i k}^{2 M}+X_{i k}^{2 R}\right)-I_{t k}^{2 S} \leq s_{t k}^{2} \\
& 1 \leq t \leq T, 1 \leq k \leq t, \\
& -\sum_{i=1}^{t}\left(X_{i 0}^{R}+X_{i 0}^{D}\right)-\sum_{i=1}^{t} \sum_{k=1}^{i-1}\left(\bar{d}_{k} X_{i k}^{1 R}+\bar{r}_{k} X_{i k}^{2 R}+\bar{d}_{k} X_{i k}^{1 D}+\bar{r}_{k} X_{i k}^{2 D}\right) \\
& -\sum_{k=1}^{t-1}\left(\hat{d}_{k} v_{t k}^{1}+\hat{r}_{k} v_{t k}^{2}\right) \geq-I_{0}^{R}-\sum_{i=1}^{t} \bar{r}_{i}+\hat{r}_{t} \quad 1 \leq t \leq T \text {, } \\
& -v_{t k}^{1} \leq \sum_{i=k+1}^{t}\left(X_{i k}^{1 R}+X_{i k}^{1 D}\right) \leq v_{t k}^{1} \quad 1 \leq t \leq T, 1 \leq k \leq t-1,
\end{aligned}
$$


Production Planning with Remanufacturing under Uncertain Demand and Returns 285

$$
\begin{array}{lr}
-v_{t k}^{2} \leq 1-\sum_{i=k+1}^{t}\left(X_{i k}^{2 R}+X_{i k}^{2 D}\right) \leq v_{t k}^{2} \quad 1 \leq t \leq T, 1 \leq k \leq t-1, \\
X_{t 0}^{M}+\sum_{k=1}^{t-1}\left(\bar{d}_{k} X_{t k}^{1 M}+\bar{r}_{k} X_{t k}^{2 M}-\hat{d}_{k} u_{t k}^{1 M}-\hat{r}_{k} u_{t k}^{2 M}\right) \geq 0 & 1 \leq t \leq T, \\
-u_{t k}^{1 M} \leq X_{t k}^{1 M} \leq u_{t k}^{1 M} & 1 \leq t \leq T, 1 \leq k \leq t-1, \\
-u_{t k}^{2 M} \leq X_{t k}^{2 M} \leq u_{t k}^{2 M} & 1 \leq t \leq T, 1 \leq k \leq t-1, \\
X_{t 0}^{R}+\sum_{k=1}^{t-1}\left(\bar{d}_{k} X_{t k}^{1 R}+\bar{r}_{k} X_{t k}^{2 R}-\hat{d}_{k} u_{t k}^{1 R}-\hat{r}_{k} u_{t k}^{2 R}\right) \geq 0 \quad 1 \leq t \leq T, \\
-u_{t k}^{1 R} \leq X_{t k}^{1 R} \leq u_{t k}^{1 R} & 1 \leq t \leq T, 1 \leq k \leq t-1, \\
-u_{t k}^{2 R} \leq X_{t k}^{2 R} \leq u_{t k}^{2 R} & 1 \leq t \leq T, 1 \leq k \leq t-1, \\
X_{t 0}^{D}+\sum_{k=1}^{t-1}\left(\bar{d}_{k} X_{t k}^{1 D}+\bar{r}_{k} X_{t k}^{2 D}-\hat{d}_{k} u_{t k}^{1 D}-\hat{r}_{k} u_{t k}^{2 D}\right) \geq 0 \quad 1 \leq t \leq T, \\
-u_{t k}^{1 D} \leq X_{t k}^{1 D} \leq u_{t k}^{1 D} & 1 \leq t \leq T, 1 \leq k \leq t-1, \\
-u_{t k}^{2 D} \leq X_{t k}^{2 D} \leq u_{t k}^{2 D} & 1 \leq t \leq T, 1 \leq k \leq t-1,
\end{array}
$$

where $\tilde{c}_{t}^{R}=c^{R}-(T-t+1) h^{R}$, and $\tilde{c}_{t}^{D}=c^{D}-(T-t+1) h^{R}$.

Note that, unlike the model of Wei et al. (2011), the proposed robust model will yield a production-disposal policy that adapts the productiondisposal quantities based on the realization of demand and returns. The production-disposal policy, which will be feasible regardless of the realization of demand and returns, is determined by the realized demand and returns, and by the values of the $X_{t 0}^{M}, X_{t k}^{1 M}, X_{t k}^{2 M}, X_{t 0}^{R}, X_{t k}^{1 R}, X_{t k}^{2 R}, X_{t 0}^{D}, X_{t k}^{1 D}, X_{t k}^{2 D}$ variables for $1 \leq t \leq T, 1 \leq k \leq t-1$, that are found by solving AARM. For example, the quantity of serviceable products that are manufactured in period 2 (i.e., $X_{2}^{M}$ ) is determined by $X_{20}^{M}+d_{1} X_{21}^{1 M}+r_{1} X_{21}^{2 M}$, where $d_{1}$ and $r_{1}$ are the realized demand and quantity returned in period 1 respectively, and $X_{20}^{M}, X_{21}^{1 M}$, and $X_{21}^{2 M}$ are found by solving AARM at time 0 .

\section{COMPUTATIONAL RESULTS}

We have performed computational experiments on test instances in order to assess the average-case performance of solutions yielded by the BURM and AARM. The average-case performance of models is assessed by considering the average performance of the solution yielded by each model for an instance over a given number of simulations of realized demand and returns. All these models were solved by CPLEX 12.5 with its default settings, and all computational 
experiments have been performed on a Dell T7500 Precision Workstation running under Windows 7.

For the computational experiments, we have generated test instances using settings similar to those used by Wei et al. (2011) as follows. The length of the planning horizon is set to 20 . The initial inventory levels of serviceable products and returned products are set to zero (i.e., $I_{0}^{S}=I_{0}^{R}=0$ ). The unit manufacturing, remanufacturing and disposing costs are taken as $c^{M}=7$, $c^{R}=4, c^{D}=2$, respectively. As assumed by Wei et al., (2011): $h^{R}=4 \leq$ $h^{S}=5$. The unit backlogging cost is set to $b \in\{3,4,5,6\}$ in order to ensure all possible relations (i.e., less than, equal, or greater than) between $h^{R}$ and $b$, and $h^{S}$ and $b$. The uncertain demand has a mean $\bar{d}_{t} \in\{18,20,22\}$ and a standard deviation $\sigma \in\{2,4\}$. In our RO framework, the uncertain demand $d_{t}$ can take any value from the interval $\left[\bar{d}_{t}-2 \sigma, \bar{d}_{t}+2 \sigma\right]$, i.e., $\hat{d}_{t}=2 \sigma$. Similarly, the uncertain returns have a mean $\bar{r}_{t} \in\{14,16,18\}$ and a standard deviation $\sigma \in\{2,4\}$. As a result, the uncertain return $r_{t}$ can take any value from the interval $\left[\bar{r}_{t}-2 \sigma, \bar{r}_{t}+2 \sigma\right]$, i.e., $\hat{r}_{t}=2 \sigma$. Thus, combining all parameter settings we have generated 72 instances for the average-case performance assessment, in total. While the budgets of uncertainty $\Gamma_{t}^{d}$ are generated such that the probability of infeasibility at constraints (2) and (3) for $t$ is less than 5\%, the budgets of uncertainty $\Gamma_{t}^{r}$ are generated such that the probability of infeasibility at constraint (4) for $t$ is less than $0.1 \%$. The probability of infeasibility at constraint (4) for $t$ is set to such a low number because infeasibility at constraints (4) indicates that an infeasible solution is obtained by solving BURM. On the other hand, the probability of infeasibility at constraints (2) and (3) for $t$ is set to 5\% because the probability of infeasibility at constraints (2) and (3) for $t$ does not affect the feasibility of the solution obtained by solving BURM while that probability affects the cost of solution obtained. To estimate the average performance of solutions provided by the different models, we generate 100 simulations of realized demand and realized returns (see e.g. BenTal et al., 2004) for each instance. In particular, realized demand and returns are generated as uniformly distributed from the interval $\left[\bar{d}_{t}-2 \sigma, \bar{d}_{t}+2 \sigma\right]$ and $\left[\bar{r}_{t}-2 \sigma, \bar{r}_{t}+2 \sigma\right]$, respectively.

We have compared the practical performances of BURM and AARM against realized demand and returns and reported the results in Table 1 where columns 1-5 show the unit backlogging cost of serviceable products, the standard deviation value of demand and returns, the mean demand and returns values, and the relative improvement brought by the AARM over BURM (Imp\%), respectively. Columns 6 and 7 indicate the average objective function value and the standard deviation of the average objective function values, given by the optimal solution of the BURM against 100 simulations of realized 
Production Planning with Remanufacturing under Uncertain Demand and Returns 287

demand and returns. Columns 8 and 9 are for the AARM and have the same meanings as 6 and 7 . The key results of Table 1 are as follows:

- The AARM performs significantly better than the BURM on all test instances with an average improvement of $54.8 \%$.

- As depicted by the columns 7 and 9, AARM yields much more stable results over different realizations of demand and returns than BURM does.

- As the uncertainty of demand and returns increases (i.e., when $\sigma$ increases), the improvement brought by AARM over BURM increases as well.

- As the mean demand increases, the improvement brought by AARM over BURM decreases in general.

- The improvement brought by AARM over BURM decreases (resp. increases) when the mean return value increases from 14 to 16 (resp. from 16 to 18).

We have also provided a graphical display of Imp\% values for different $b$ values in Figure 1 in order to see how Imp\% values are affected by different parameters. First of all, it seems that the percentage improvement brought by AARM over BURM does not seem to be affected by the unit inventory backlogging cost. It is easy to observe that when the variability of uncertain demand and returns is larger (right-half of the figure), the percentage improvement brought by AARM over BURM is larger too.

Figure 1. The Percentage Improvement brought by AARM over BURM for Different $b$. 


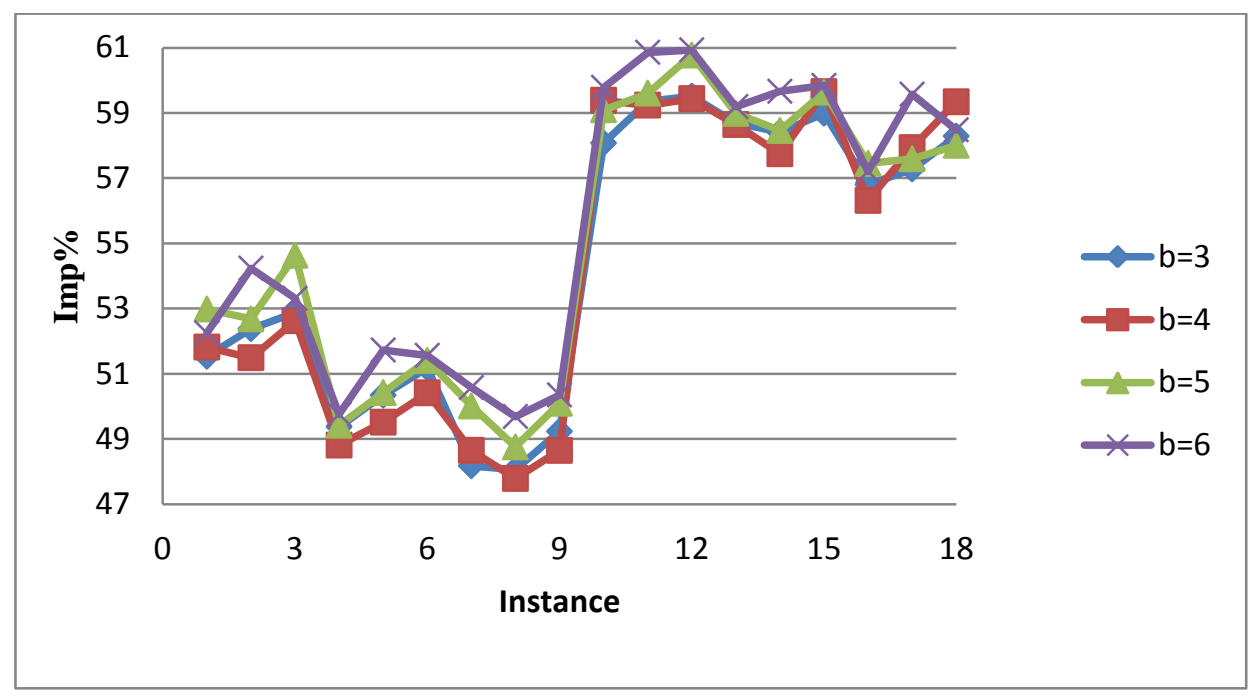

Table 1. The Average-Case Performances of BURM and AARM.

\begin{tabular}{|c|c|c|c|c|c|c|c|c|}
\hline \multirow[b]{2}{*}{ B } & \multirow[b]{2}{*}{$\sigma$} & \multirow[b]{2}{*}{$\overline{\boldsymbol{d}}_{\boldsymbol{t}}$} & \multirow[b]{2}{*}{$\overline{\boldsymbol{r}}_{\boldsymbol{t}}$} & \multirow[b]{2}{*}{ Imp\% } & \multicolumn{2}{|c|}{ BURM } & \multicolumn{2}{|c|}{ AARM } \\
\hline & & & & & Average & $\begin{array}{l}\text { Standard } \\
\text { Dev. }\end{array}$ & Average & $\begin{array}{l}\text { Standard } \\
\text { Dev. }\end{array}$ \\
\hline 3 & 2 & 18 & 14 & 51.5 & 5121.1 & 648.4 & 2482.2 & 24.4 \\
\hline 3 & 2 & 18 & 16 & 52.4 & 5012.7 & 592.8 & 2387.2 & 26.3 \\
\hline 3 & 2 & 18 & 18 & 52.9 & 4971.8 & 510.0 & 2342.8 & 23.2 \\
\hline 3 & 2 & 20 & 14 & 49.4 & 5439.6 & 523.3 & 2754.3 & 19.3 \\
\hline 3 & 2 & 20 & 16 & 50.3 & 5378.3 & 584.0 & 2671.0 & 19.1 \\
\hline 3 & 2 & 20 & 18 & 51.2 & 5248.1 & 591.3 & 2562.3 & 23.5 \\
\hline 3 & 2 & 22 & 14 & 48.2 & 5698.3 & 630.3 & 2953.4 & 31.4 \\
\hline 3 & 2 & 22 & 16 & 48.1 & 5633.5 & 532.5 & 2926.3 & 25.0 \\
\hline 3 & 2 & 22 & 18 & 49.2 & 5537.0 & 600.6 & 2811.3 & 24.6 \\
\hline 3 & 4 & 18 & 14 & 58.1 & 8569.0 & 1145.4 & 3592.7 & 75.1 \\
\hline 3 & 4 & 18 & 16 & 59.4 & 8670.0 & 960.5 & 3523.8 & 70.4 \\
\hline 3 & 4 & 18 & 18 & 59.5 & 8222.7 & 1035.2 & 3329.3 & 77.0 \\
\hline 3 & 4 & 20 & 14 & 58.7 & 9093.1 & 964.4 & 3756.3 & 73.8 \\
\hline 3 & 4 & 20 & 16 & 58.4 & 8773.2 & 1056.2 & 3649.0 & 60.4 \\
\hline 3 & 4 & 20 & 18 & 59.0 & 8653.0 & 1082.9 & 3548.8 & 63.7 \\
\hline 3 & 4 & 22 & 14 & 56.8 & 9173.6 & 1103.5 & 3963.5 & 60.6 \\
\hline 3 & 4 & 22 & 16 & 57.3 & 9008.5 & 1143.0 & 3848.7 & 53.3 \\
\hline 3 & 4 & 22 & 18 & 58.3 & 8933.6 & 1094.0 & 3726.5 & 61.2 \\
\hline 4 & 2 & 18 & 14 & 51.8 & 5277.9 & 603.7 & 2543.2 & 17.0 \\
\hline 4 & 2 & 18 & 16 & 51.5 & 5066.4 & 532.0 & 2457.8 & 21.9 \\
\hline
\end{tabular}


Production Planning with Remanufacturing under Uncertain Demand and Returns 289

\begin{tabular}{|l|l|l|l|l|r|r|r|r|}
4 & 2 & 18 & 18 & 52.6 & 4998.4 & 625.1 & 2368.0 & 25.8 \\
\hline 4 & 2 & 20 & 14 & 48.8 & 5498.3 & 576.7 & 2814.8 & 16.6 \\
\hline 4 & 2 & 20 & 16 & 49.5 & 5343.2 & 520.8 & 2697.8 & 18.0 \\
\hline 4 & 2 & 20 & 18 & 50.4 & 5262.0 & 537.7 & 2609.4 & 19.7 \\
\hline 4 & 2 & 22 & 14 & 48.6 & 5764.7 & 566.4 & 2960.4 & 25.2 \\
\hline 4 & 2 & 22 & 16 & 47.8 & 5686.9 & 600.1 & 2969.5 & 16.0 \\
\hline 4 & 2 & 22 & 18 & 48.6 & 5551.5 & 575.6 & 2851.1 & 18.5 \\
\hline 4 & 4 & 18 & 14 & 59.4 & 8868.4 & 1074.1 & 3601.7 & 75.8 \\
\hline 4 & 4 & 18 & 16 & 59.2 & 8750.2 & 1117.4 & 3566.9 & 65.9 \\
\hline 4 & 4 & 18 & 18 & 59.4 & 8583.1 & 1183.6 & 3481.6 & 69.3 \\
\hline 4 & 4 & 20 & 14 & 58.6 & 9270.4 & 1144.3 & 3834.2 & 54.4 \\
\hline 4 & 4 & 20 & 16 & 57.7 & 8917.1 & 1093.1 & 3768.0 & 57.5 \\
\hline 4 & 4 & 20 & 18 & 59.7 & 9041.8 & 1241.0 & 3648.3 & 52.1 \\
\hline 4 & 4 & 22 & 14 & 56.3 & 9252.7 & 1109.8 & 4042.3 & 50.6 \\
\hline 4 & 4 & 22 & 16 & 57.9 & 9335.4 & 1210.8 & 3927.2 & 49.5 \\
\hline 4 & 4 & 22 & 18 & 59.3 & 9420.8 & 1044.5 & 3830.7 & 44.9 \\
\hline
\end{tabular}

Table 1. Continued

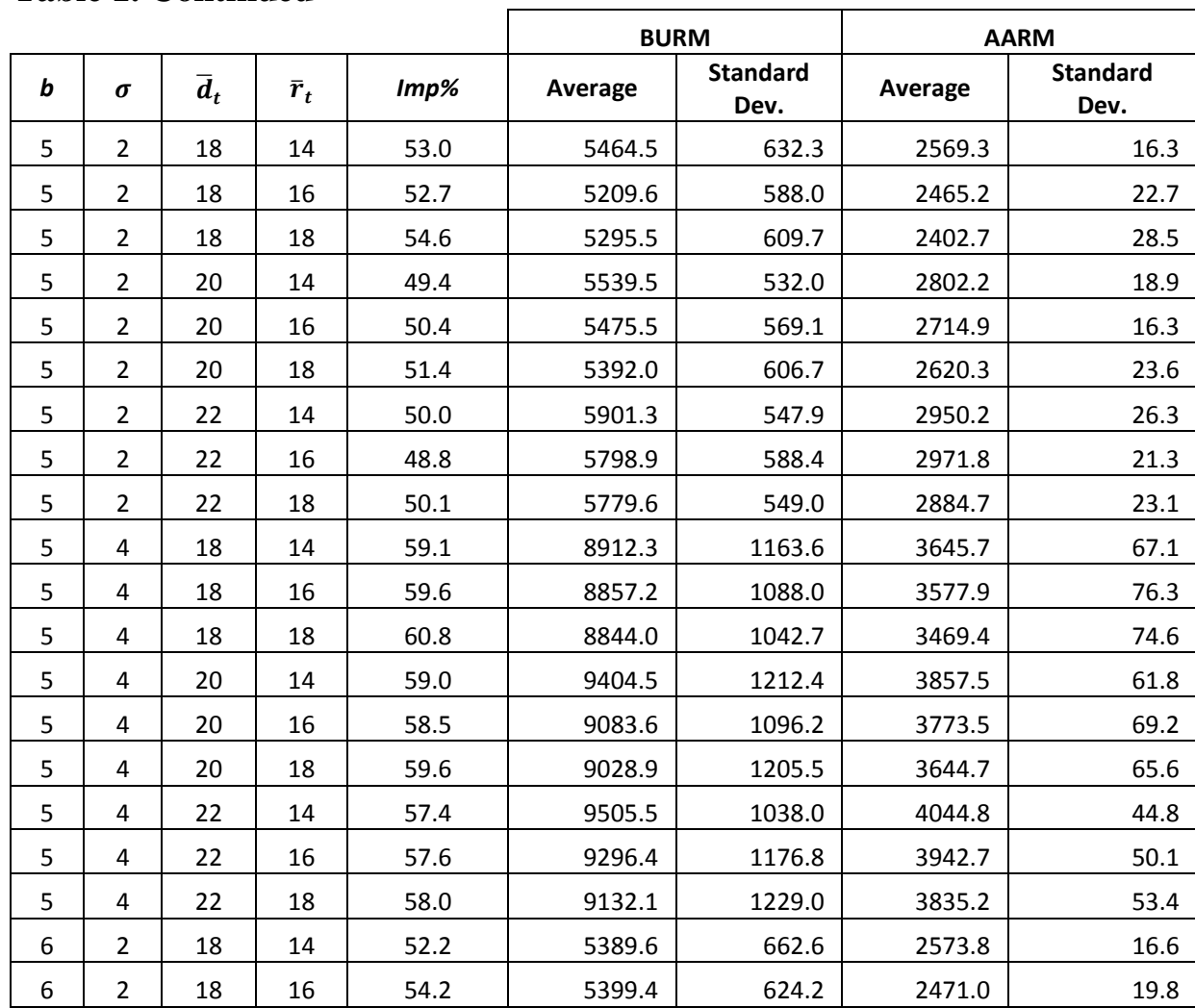




\begin{tabular}{|l|l|l|l|l|r|r|r|r|}
6 & 2 & 18 & 18 & 53.3 & 5167.9 & 631.3 & 2412.9 & 25.3 \\
\hline 6 & 2 & 20 & 14 & 49.8 & 5586.9 & 575.8 & 2807.3 & 21.5 \\
\hline 6 & 2 & 20 & 16 & 51.7 & 5626.9 & 600.1 & 2716.4 & 19.9 \\
\hline 6 & 2 & 20 & 18 & 51.6 & 5471.8 & 638.1 & 2650.5 & 23.4 \\
\hline 6 & 2 & 22 & 14 & 50.6 & 6062.4 & 590.5 & 2996.5 & 28.2 \\
\hline 6 & 2 & 22 & 16 & 49.7 & 5900.0 & 564.4 & 2968.6 & 20.9 \\
\hline 6 & 2 & 22 & 18 & 50.3 & 5798.6 & 618.7 & 2879.2 & 19.3 \\
\hline 6 & 4 & 18 & 14 & 59.8 & 9213.1 & 1171.4 & 3705.3 & 68.3 \\
\hline 6 & 4 & 18 & 16 & 60.9 & 9202.5 & 1107.5 & 3602.0 & 77.5 \\
\hline 6 & 4 & 18 & 18 & 60.9 & 8900.1 & 1263.0 & 3477.1 & 81.0 \\
\hline 6 & 4 & 20 & 14 & 59.2 & 9478.6 & 1236.9 & 3868.0 & 63.1 \\
\hline 6 & 4 & 20 & 16 & 59.7 & 9293.8 & 1181.2 & 3749.0 & 63.6 \\
\hline 6 & 4 & 20 & 18 & 59.8 & 9205.0 & 1114.9 & 3696.7 & 59.7 \\
\hline 6 & 4 & 22 & 14 & 57.2 & 9507.2 & 1207.6 & 4068.3 & 47.5 \\
\hline 6 & 4 & 22 & 16 & 59.6 & 9770.2 & 1127.3 & 3949.7 & 51.8 \\
\hline 6 & 4 & 22 & 18 & 58.5 & 9304.7 & 1088.6 & 3863.4 & 53.2 \\
\hline
\end{tabular}

In order to understand why AARM performs much better than BURM, we have analyzed the average cost compositions yielded by both models. We present the average cost compositions over all instances in Table 2, where IHC$\mathrm{S}$ (resp. IBC-S) denotes the inventory holding (resp. backlogging) cost percentage for serviceable products, IHC-R the inventory holding cost percentage for the returned products, MC the variable manufacturing cost percentage, $\mathrm{RC}$ the variable remanufacturing cost percentage, and DC the variable disposal cost percentage.

Table 2. Average Cost Compositions Yielded by AARM and BURM

\begin{tabular}{|l|r|r|r|r|r|r|}
\hline Model & \multicolumn{1}{|c|}{ IHC-S } & \multicolumn{1}{c|}{ IBC-S } & \multicolumn{1}{c|}{ IHC-R } & \multicolumn{1}{c|}{ MC } & \multicolumn{1}{c|}{ RC } & \multicolumn{1}{c|}{ DC } \\
\hline AARM & 14.3 & 1.1 & 14.7 & 34.2 & 30.9 & 4.7 \\
\hline BURM & 4.5 & 6.8 & 59.3 & 15.3 & 14.1 & 0.0 \\
\hline
\end{tabular}

It is easy to observe from Table 2 that unlike AARM, the main cost item of BURM is by far the inventory holding cost for returned products, which is due to the requirement to ensure feasibility for the returned products inventory. On the other hand, the major cost items of AARM are variable manufacturing and remanufacturing costs.

Figure 2. Average Quantities that are Obtained by AARM and BURM on Instances with $\boldsymbol{b}=\mathbf{3}$. 


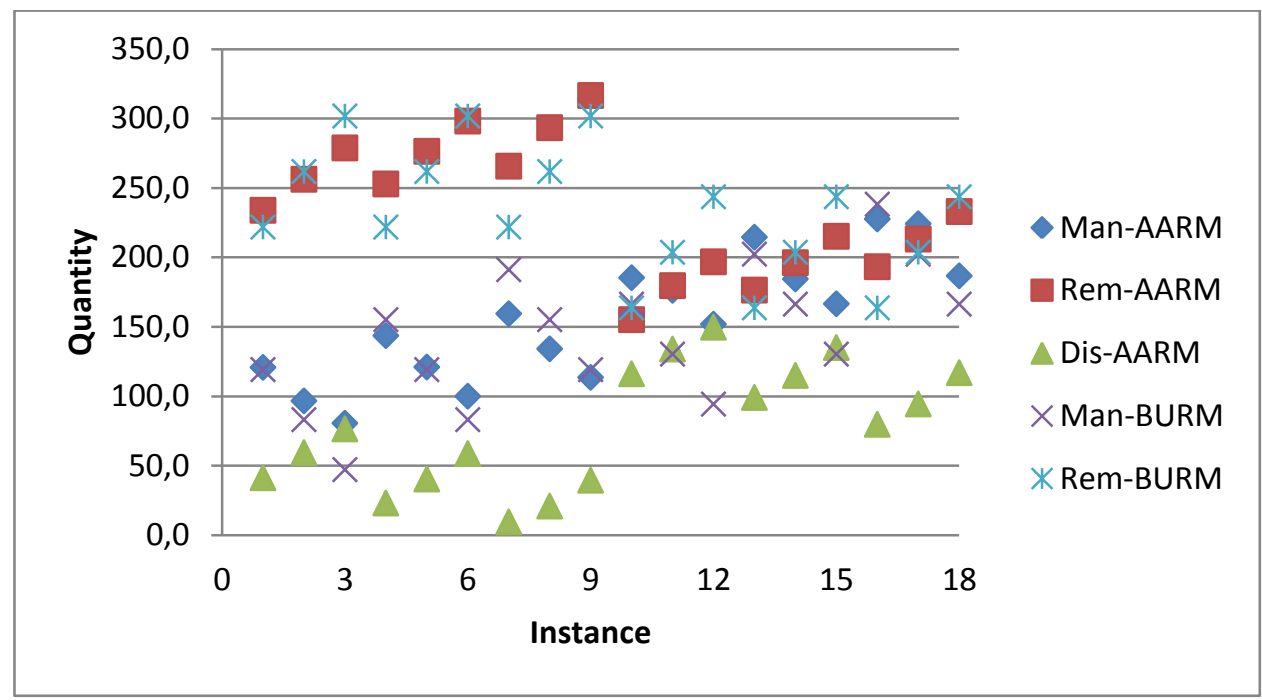

Next, we analyze the solutions generated by AARM and BURM. Figure 2 shows the average manufacturing, remanufacturing and disposal quantities that are obtained by AARM and BURM on instances with $b=3$ (i.e., first 18 instances in Table 1). Because the results on instances with $b=4,5$, and 6 are quite similar to the results on instances with $b=3$, we present only the results on instances with $b=3$. In Figure 2, Man-AARM, Rem-AARM, and DisAARM denote the average manufacturing, remanufacturing and disposal quantities yielded by AARM, respectively, while Man-BURM and Rem-BURM denote the average manufacturing and remanufacturing quantities generated by BURM, respectively. Note that as the average disposal quantities generated by BURM were always zero, they are not presented in Figure 2. Key insights that are obtained regarding the average quantities yielded by AARM and BURM are as follows:

- In all instances, the total of average remanufacturing and disposal quantities generated by AARM was greater than that generated by BURM. This indicates that BURM is more conservative than AARM in ensuring feasibility of the returned products inventory which results in higher inventory holding costs of returned products for BURM (also shown by Table 2).

- For AARM, as the mean quantity of returned products increases, the average remanufacturing and disposal quantities increased whereas the average manufacturing quantities decreased. The same observation is valid for quantities obtained by BURM except that the disposal quantities yielded by BURM were always zero. 
- As the mean demand increases, the average manufacturing and remanufacturing quantities increased for both AARM and BURM. Contrary to the zero disposal quantities yielded by BURM, the average disposal quantities yielded by AARM decreased as the mean demand increases.

- For AARM, as the uncertainty of demand and returns increases, the average manufacturing and disposal quantities increased whereas the average remanufacturing quantities decreased. We have the same comment for BURM except for the zero disposal quantities.

\section{CONCLUSION}

By developing a new robust LP model and a policy, this study contributes to the state of knowledge on how producers should simultaneously plan for their remanufacturing and regular manufacturing under demand and return uncertainty. It has been shown that the robust LP model existing in the literature has flaws, and then the flaws in the model have been corrected. It has empirically been shown that the new robust LP model yields superior policies and results for the production planning problem with remanufacturing under demand and returns uncertainty than the model existing in the literature does. Thus, the researchers and decision makers considering such problems are presented a powerful decision making tool.

\section{REFERENCES}

Akçalı, E., S. Çetinkaya (2011) "Quantitative Models for Inventory and Production Planning in Closed-loop Supply Chains", International Journal of Production Research, 49(8), 2373-2407.

Ben-Tal, A., B. Golany, A. Nemirovski, J-P. Vial (2005) "Retailer-Supplier Flexible Commitments Contracts: A Robust Optimization Approach", Manufacturing \& Service Operations Management, 7(3), 248-271.

Ben-Tal, A., B. Golany, S. Shtern (2009) "Robust Multi-Echelon Multi-Period Inventory Control”, European Journal of Operarional Research, 199(3), 922-935.

Ben-Tal, A., A. Goryashko, E. Guslitzer, A. Nemirovski (2004) “Adjustable Robust Solutions of Uncertain Linear Programs", Mathematical Programming, Series A, 99(2), 351-376.

Ben-Tal, A., A. Nemirovski (1998) "Robust Convex Optimization", Mathematics of Operations Research, 23(4), 769-805. 
Production Planning with Remanufacturing under Uncertain Demand and Returns 293

Ben-Tal, A., A. Nemirovski (1999) "Robust Solutions of Uncertain Linear Programs", Operations Research Letters, 25(1), 1-13.

Ben-Tal, A., A. Nemirovski (2000) "Robust Solutions of Linear Programming Problems Contaminated with Uncertain Data", Mathematical Programming, Series A, $88(3), 411-424$.

Bertsimas, D., M. Sim (2003) "Robust Discrete Optimization and Network Flows", Mathematical Programming, Series B, 98(1), 49-71.

Bertsimas, D., M. Sim (2004) “The Price of Robustness”, Operations Research, 52(1), $35-53$.

Bertsimas, D., A. Thiele (2006) "A Robust Optimization Approach to Inventory Theory”, Operations Research, 54(1), 150-168.

Bertsimas, D., D.A. Iancu, P.A. Parrilo (2010) "Optimality of Affine Policies in Multistage Robust Optimization", Mathematics of Operations Research, 35(2), 363-394.

Bienstock, D., N. Özbay (2008) "Computing Robust Basestock Levels", Discrete Optimization, 5(2), 389-414.

Chunfa, L., H. Linna, L. Li, W. Qiang (2011) "Robust Single Echelon Multi Period Inventory of Eco-Industrial Symbiotic Network with Uncertain Returns and Demand", International Conference on Management Science and Industrial Engineering 2011, 1209-1213.

Helmrich, M.J.R., R. Jans, W. van den Heuvel, A.P.M. Wagelmans (2014) "Economic lot-sizing with Remanufacturing: Complexity and Efficient Formulations", IIE Transactions, 46(1), 67-86.

Li, X., F. Baki, P.Tian, B.A. Chaouch (2014) "A Robust Block-Chain Based Tabu Search Algorithm for the Dynamic Lot Sizing Problem with Product Returns and Remanufacturing", OMEGA, 42(1), 75-87.

Naeem, M.A., D.J. Dias, R. Tibrewal, P.C. Chang, M.K. Tiwari (2013) "Production Planning Optimization for Manufacturing and Remanufacturing System in Stochastic Environment", Journal of Intelligent Manufacturing, 24(4), 717 728.

See, C.-T., M. Sim (2010) "Robust Approximation to Multiperiod Inventory Management", Operations Research, 58(3), 583-594.

Shi, J., G. Zhang, J. Sha (2011) "Optimal Production Planning for a Multi-Product Closed Loop System with Uncertain Demand and Return", Computers \& Operations Research, 38(3), 641-650. 
Wei, C., Y. Li, X. Cai (2011) "Robust Optimal Policies of Production and Inventory with Uncertain Returns and Demand", International Journal of Production Economics, 134(2), 357-367.

\section{APPENDIX DERIVATION OF AARM}

Here we describe in detail how the affinely adjustable robust model is derived.

(a) The objective function (25), constraints (26) and (27): First note that

$\sum_{t=1}^{T} \sum_{i=1}^{t}-h^{R} X_{i}^{R}=\sum_{i=1}^{T} \sum_{t=i}^{T}-h^{R} X_{i}^{R}=-\sum_{i=1}^{T} X_{i}^{R} \sum_{t=i}^{T} h^{R}$

$=-\sum_{i=1}^{T}(T-i+1) h^{R} X_{i}^{R}$.

Similarly,

$\sum_{t=1}^{T} \sum_{i=1}^{t}-h^{R} X_{i}^{D}=-\sum_{i=1}^{T}(T-i+1) h^{R} X_{i}^{D}$

Using (46) and (47), the objective function (1) can be rewritten as

$T h^{R} I_{0}^{R}+h^{R} \sum_{t=1}^{T} \sum_{i=1}^{t} r_{i}-\sum_{i=1}^{T}(T-i+1) h^{R} X_{i}^{R}-\sum_{i=1}^{T}(T-i+1) h^{R} X_{i}^{D}$

$+\sum_{t=1}^{T}\left[I_{t}^{S}+c^{M} X_{t}^{M}+c^{R} X_{t}^{R}+c^{D} X_{t}^{D}\right]$

$=T h^{R} I_{0}^{R}+h^{R} \sum_{t=1}^{T} \sum_{i=1}^{t} r_{i}+\sum_{t=1}^{T}\left[I_{t}^{S}+c^{M} X_{t}^{M}+\tilde{c}_{t}^{R} X_{t}^{R}+\tilde{c}_{t}^{D} X_{t}^{D}\right]$,

where $\tilde{c}_{t}^{R}=c^{R}-(T-t+1) h^{R}$ and $\tilde{c}_{t}^{D}=c^{D}-(T-t+1) h^{R}$.

Substituting the right-hand sides of (18)-(21) in place of $I_{t}^{S}, X_{t}^{M}, X_{t}^{R}$ and $X_{t}^{D}$ variables in (48), respectively, gives

$$
\begin{aligned}
& \sum_{t=1}^{T}\left(I_{t 0}^{S}+\sum_{k=1}^{t}\left(d_{k} I_{t k}^{1 S}+r_{k} I_{t k}^{2 S}\right)\right)+c^{M} \sum_{t=1}^{T}\left(X_{t 0}^{M}+\sum_{k=1}^{t-1}\left(d_{k} X_{t k}^{1 M}+r_{k} X_{t k}^{2 M}\right)\right) \\
& +\sum_{t=1}^{T} \tilde{c}_{t}^{R}\left(X_{t 0}^{R}+\sum_{k=1}^{t-1}\left(d_{k} X_{t k}^{1 R}+r_{k} X_{t k}^{2 R}\right)\right)+\sum_{t=1}^{T} \tilde{c}_{t}^{D}\left(X_{t 0}^{D}+\sum_{k=1}^{t-1}\left(d_{k} X_{t k}^{1 D}+r_{k} X_{t k}^{2 D}\right)\right) \\
& +T h^{R} I_{0}^{R}+h^{R} \sum_{t=1}^{T} \sum_{k=1}^{t} r_{k} .
\end{aligned}
$$

Using the equivalences (i.e., changing the summation bounds) $\sum_{t=1}^{T} \sum_{k=1}^{t} Y=\sum_{k=1}^{T} \sum_{t=k}^{T} Y$ and $\sum_{t=1}^{T} \sum_{k=1}^{t-1} Y=\sum_{k=1}^{T-1} \sum_{t=k+1}^{T} Y$ for any $Y,(49)$ can be rewritten as

$$
\begin{aligned}
& \sum_{k=1}^{T} \sum_{t=k}^{T} d_{k} I_{t k}^{1 S}+\sum_{k=1}^{T-1} \sum_{t=k+1}^{T}\left(c^{M} d_{k} X_{t k}^{1 M}+\tilde{c}_{t}^{R} d_{k} X_{t k}^{1 R}+\tilde{c}_{t}^{D} d_{k} X_{t k}^{1 D}\right) \\
& +\sum_{k=1}^{T} \sum_{t=k}^{T} r_{k} I_{t k}^{2 S}+\sum_{k=1}^{T-1} \sum_{t=k+1}^{T}\left(c^{M} r_{k} X_{t k}^{2 M}+\tilde{c}_{t}^{R} r_{k} X_{t k}^{2 R}+\tilde{c}_{t}^{D} r_{k} X_{t k}^{2 D}\right) \\
& +\sum_{t=1}^{T}\left(I_{t 0}^{S}+c^{M} X_{t 0}^{M}+\tilde{c}_{t}^{R} X_{t 0}^{R}+\tilde{c}_{t}^{D} X_{t 0}^{D}\right)+T h^{R} I_{0}^{R}+h^{R} \sum_{k=1}^{T} \sum_{t=k}^{T} r_{k} \\
& =d_{T} I_{T T}^{1 S}+\sum_{k=1}^{T-1}\left[d_{k} I_{k k}^{1 S}+\sum_{t=k+1}^{T}\left(d_{k} I_{t k}^{1 S}+c^{M} d_{k} X_{t k}^{1 M}+\tilde{c}_{t}^{R} d_{k} X_{t k}^{1 R}+\tilde{c}_{t}^{D} d_{k} X_{t k}^{1 D}\right)\right] \\
& +r_{T} I_{T T}^{2 S}+\sum_{k=1}^{T-1}\left[r_{k} I_{k k}^{2 S}+\sum_{t=k+1}^{T}\left(r_{k} I_{t k}^{2 S}+c^{M} r_{k} X_{t k}^{2 M}+\tilde{c}_{t}^{R} r_{k} X_{t k}^{2 R}+\tilde{c}_{t}^{D} r_{k} X_{t k}^{2 D}\right)\right. \\
& +\sum_{t=1}^{T}\left(I_{t 0}^{S}+c^{M} X_{t 0}^{M}+\tilde{c}_{t}^{R} X_{t 0}^{R}+\tilde{c}_{t}^{D} X_{t 0}^{D}\right)+T h^{R} I_{0}^{R}+\sum_{k=1}^{T} h^{R}(T-k+1) r_{k} .
\end{aligned}
$$

Aggregating terms involving $d_{k}$ and $r_{k}$ in (50), one obtains

$$
d_{T} I_{T T}^{1 S}+\sum_{k=1}^{T-1} d_{k}\left[I_{k k}^{1 S}+\sum_{t=k+1}^{T}\left(I_{t k}^{1 S}+c^{M} X_{t k}^{1 M}+\tilde{c}_{t}^{R} X_{t k}^{1 R}+\tilde{c}_{t}^{D} X_{t k}^{1 D}\right)\right]+r_{T}\left(I_{T T}^{2 S}+h^{R}\right)
$$


$+\sum_{k=1}^{T-1} r_{k}\left[I_{k k}^{2 S}+\sum_{t=k+1}^{T}\left(I_{t k}^{2 S}+c^{M} X_{t k}^{2 M}+\tilde{c}_{t}^{R} X_{t k}^{2 R}+\tilde{c}_{t}^{D} X_{t k}^{2 D}\right)+h^{R}(T-k+1)\right]$

$+\sum_{t=1}^{T}\left(I_{t 0}^{S}+c^{M} X_{t 0}^{M}+\tilde{c}_{t}^{R} X_{t 0}^{R}+\tilde{c}_{t}^{D} X_{t 0}^{D}\right)+T h^{R} I_{0}^{R}$.

Note that the objective function (51) can be rewritten as $\operatorname{Min}\{Z: Z \geq A\}$ where A denotes (51). Using the methodology described in Section 3 to reformulate (22) as (23) and (24), one can equivalently reformulate (51) as

$Z \geq \bar{d}_{T} I_{T T}^{1 S}+\sum_{k=1}^{T-1} \bar{d}_{k}\left[I_{k k}^{1 S}+\sum_{t=k+1}^{T}\left(I_{t k}^{1 S}+c^{M} X_{t k}^{1 M}+\tilde{c}_{t}^{R} X_{t k}^{1 R}+\tilde{c}_{t}^{D} X_{t k}^{1 D}\right)\right]+\bar{r}_{T}\left(I_{T T}^{2 S}+h^{R}\right)$

$+\sum_{k=1}^{T-1} \bar{r}_{k}\left[I_{k k}^{2 S}+\sum_{t=k+1}^{T}\left(I_{t k}^{2 S}+c^{M} X_{t k}^{2 M}+\tilde{c}_{t}^{R} X_{t k}^{2 R}+\tilde{c}_{t}^{D} X_{t k}^{2 D}\right)+h^{R}(T-k+1)\right]$

$+\sum_{t=1}^{T}\left(I_{t 0}^{S}+c^{M} X_{t 0}^{M}+\tilde{c}_{t}^{R} X_{t 0}^{R}+\tilde{c}_{t}^{D} X_{t 0}^{D}\right)+T h^{R} I_{0}^{R}+\sum_{t=1}^{T}\left(\hat{d}_{t} p_{t}^{1}+\hat{r}_{t} p_{t}^{2}\right)$,

$-p_{k}^{1} \leq I_{k k}^{1 S}+\sum_{t=k+1}^{T}\left(I_{t k}^{1 S}+c^{M} X_{t k}^{1 M}+\tilde{c}_{t}^{R} X_{t k}^{1 R}+\tilde{c}_{t}^{D} X_{t k}^{1 D}\right) \leq p_{k}^{1} \quad 1 \leq k \leq T$,

$-p_{k}^{2} \leq I_{k k}^{2 S}+h^{R}(T-k+1)+\sum_{t=k+1}^{T}\left(I_{t k}^{2 S}+c^{M} X_{t k}^{2 M}+\tilde{c}_{t}^{r} X_{t k}^{2 R}+\tilde{c}_{t}^{d} X_{t k}^{2 D}\right) \leq p_{k}^{2}$

$1 \leq k \leq T$,

Note that $(52)-(54)$ are equivalent to $(25)-(27)$, respectively. Thus, $\sum_{t=1}^{T}\left(\hat{d}_{t} p_{t}^{1}+\hat{r}_{t} p_{t}^{2}\right)$ is the additional term due to the uncertainty in demand and returns in addition to the terms involving mean demand and returns in the objective function.

(b) Constraints (28)-(30): Substituting the right-hand sides of (18), (19) and (21) in place of $X_{t}^{M}, X_{t}^{R}$ and $I_{t}^{S}$ variables in (2), respectively, gives

$I_{t 0}^{S}+\sum_{k=1}^{t}\left(d_{k} I_{t k}^{1 S}+r_{k} I_{t k}^{2 S}\right) \geq h^{S}\left[I_{0}^{S}+\sum_{i=1}^{t}\left(X_{i 0}^{M}+\sum_{k=1}^{i-1}\left(d_{k} X_{i k}^{1 M}+r_{k} X_{i k}^{2 M}\right)+X_{i 0}^{R}+\right.\right.$ $\left.\left.\sum_{k=1}^{i-1}\left(d_{k} X_{i k}^{1 R}+r_{k} X_{i k}^{2 R}\right)-d_{i}\right)\right] \quad 1 \leq t \leq T, d \in D_{t}, r \in R_{t}$,

which can be rewritten as

$I_{t 0}^{S}+\sum_{k=1}^{t}\left(d_{k} I_{t k}^{1 S}+r_{k} I_{t k}^{2 S}\right) \geq h^{S} I_{0}^{S}+h^{S} \sum_{i=1}^{t} X_{i 0}^{M}+h^{S} \sum_{i=1}^{t} \sum_{k=1}^{i-1}\left(d_{k} X_{i k}^{1 M}+r_{k} X_{i k}^{2 M}\right)+$ $h^{S} \sum_{i=1}^{t} X_{i 0}^{R}+h^{S} \sum_{i=1}^{t} \sum_{k=1}^{i-1}\left(d_{k} X_{i k}^{1 R}+r_{k} X_{i k}^{2 R}\right)-h^{S} \sum_{i=1}^{t} d_{i} \quad 1 \leq t \leq T, d \in D_{t}, r \in R_{t}$.

Using the equivalence (i.e., changing the summation bounds) $\sum_{i=1}^{t} \sum_{k=1}^{i-1} Y=\sum_{k=1}^{t-1} \sum_{i=k+1}^{t} Y$ for any $Y$, (55) can be rewritten as

$I_{t 0}^{S}+\sum_{k=1}^{t}\left(d_{k} I_{t k}^{1 S}+r_{k} I_{t k}^{2 S}\right) \geq h^{S} I_{0}^{S}+h^{S} \sum_{i=1}^{t}\left(X_{i 0}^{M}+X_{i 0}^{R}\right)+h^{S} \sum_{k=1}^{t-1} \sum_{i=k+1}^{t}\left(d_{k} X_{i k}^{1 M}+\right.$ $\left.r_{k} X_{i k}^{2 M}\right)+h^{S} \sum_{k=1}^{t-1} \sum_{i=k+1}^{t}\left(d_{k} X_{i k}^{1 R}+r_{k} X_{i k}^{2 R}\right)-h^{S} \sum_{k=1}^{t} d_{k} \quad 1 \leq t \leq T, d \in D_{t}, r \in R_{t}$.

(56) can be recast as

$I_{t 0}^{S} \geq$

$h^{S} I_{0}^{S}+h^{S} \sum_{i=1}^{t}\left(X_{i 0}^{M}+X_{i 0}^{R}\right)-d_{t} I_{t t}^{1 S}+\sum_{k=1}^{t-1}\left[-d_{k} I_{t k}^{1 S}+h^{S} \sum_{i=k+1}^{t} d_{k} X_{i k}^{1 M}+h^{S} \sum_{i=k+1}^{t} d_{k} X_{i k}^{1 R}-\right.$

$\left.h^{S} d_{k}\right]-h^{S} d_{t}-r_{t} I_{t t}^{2 S}+\sum_{k=1}^{t-1}\left[-r_{k} I_{t k}^{2 S}+h^{S} \sum_{i=k+1}^{t} r_{k} X_{i k}^{2 M}+h^{S} \sum_{i=k+1}^{t} r_{k} X_{i k}^{2 R}\right]$ $1 \leq t \leq T, d \in D_{t}, r \in R_{t}$.

Aggregating terms involving $d_{k}$ and $r_{k}$ in (57), one obtains

$I_{t 0}^{S} \geq h^{S} I_{0}^{S}+h^{S} \sum_{i=1}^{t}\left(X_{i 0}^{M}+X_{i 0}^{R}\right)+d_{t}\left(-I_{t t}^{1 S}-h^{S}\right)+\sum_{k=1}^{t-1} d_{k}\left[-I_{t k}^{1 S}+h^{S} \sum_{i=k+1}^{t}\left(X_{i k}^{1 M}+X_{i k}^{1 R}\right)-\right.$ $\left.h^{S}\right]-r_{t} I_{t t}^{2 S}+\sum_{k=1}^{t-1} r_{k}\left[-I_{t k}^{2 S}+h^{S} \sum_{i=k+1}^{t}\left(X_{i k}^{2 M}+X_{i k}^{2 R}\right)\right] \quad 1 \leq t \leq T, d \in D_{t}, r \in R_{t}$. 
Using the methodology described in Section 3, one can equivalently reformulate (58) as

$I_{t 0}^{S} \geq h^{S} I_{0}^{S}+h^{S} \sum_{i=1}^{t}\left(X_{i 0}^{M}+X_{i 0}^{R}\right)+\bar{d}_{t}\left(-I_{t t}^{1 S}-h^{S}\right)+\sum_{k=1}^{t-1} \bar{d}_{k}\left[-I_{t k}^{1 S}+h^{S} \sum_{i=k+1}^{t}\left(X_{i k}^{1 M}+X_{i k}^{1 R}\right)-\right.$

$\left.h^{S}\right]-\bar{r}_{t} I_{t t}^{2 S}+\sum_{k=1}^{t-1} \bar{r}_{k}\left[-I_{t k}^{2 S}+h^{S} \sum_{i=k+1}^{t}\left(X_{i k}^{2 M}+X_{i k}^{2 R}\right)\right]$

$+\sum_{k=1}^{t}\left(\hat{d}_{k} n_{t k}^{1}+\hat{r}_{k} n_{t k}^{2}\right)$

$1 \leq t \leq T$

$-n_{t k}^{1} \leq-I_{t k}^{1 S}+h^{S} \sum_{i=k+1}^{t}\left(X_{i k}^{1 M}+X_{i k}^{1 R}\right)-h^{S} \leq n_{t k}^{1} \quad 1 \leq t \leq T, 1 \leq k \leq t$,

$-n_{t k}^{2} \leq-I_{t k}^{2 S}+h^{S} \sum_{i=k+1}^{t}\left(X_{i k}^{2 M}+X_{i k}^{2 R}\right) \leq n_{t k}^{2}$

$1 \leq t \leq T, 1 \leq k \leq t$

The term $\sum_{k=1}^{t}\left(\hat{d}_{k} n_{t k}^{1}+\hat{r}_{k} n_{t k}^{2}\right)$ in (59) is due to the uncertainty in demand and returns. Excluding the term $\sum_{k=1}^{t}\left(\hat{d}_{k} n_{t k}^{1}+\hat{r}_{k} n_{t k}^{2}\right)$ in (59), the rest is equivalent to the expression in (55) with demand and returns replaced with their estimated means. Thus, (59) is equivalent to (28). The constraints (60) and (61) are the same as (29) and (30), respectively.

(c) Constraints (31)-(33): Similar to part (b).

(d) Constraints (34)-(36): Substituting the right-hand sides of (19) and (20) in place of $X_{t}^{R}$ and $X_{t}^{D}$ variables, respectively, in (4) yields

$I_{0}^{R}+\sum_{i=1}^{t}\left(r_{i}-X_{i 0}^{R}-\sum_{k=1}^{i-1}\left(d_{k} X_{i k}^{1 R}+r_{k} X_{i k}^{2 R}\right)-X_{i 0}^{D}-\sum_{k=1}^{i-1}\left(d_{k} X_{i k}^{1 D}+r_{k} X_{i k}^{2 D}\right)\right) \geq 0$ $1 \leq t \leq T, d \in D_{t-1}, r \in R_{t}$,

Using the equivalence $\sum_{i=1}^{t} \sum_{k=1}^{i-1} Y=\sum_{k=1}^{t-1} \sum_{i=k+1}^{t} Y$ for any $Y$, (62) can be rewritten as

$$
\begin{gathered}
I_{0}^{R}-\sum_{i=1}^{t}\left(X_{i 0}^{R}+X_{i 0}^{D}\right)+\sum_{i=1}^{t} r_{i}-\sum_{k=1}^{t-1} \sum_{i=k+1}^{t}\left(d_{k} X_{i k}^{1 R}+r_{k} X_{i k}^{2 R}+d_{k} X_{i k}^{1 D}+r_{k} X_{i k}^{2 D}\right) \geq 0 \\
1 \leq t \leq T, d \in D_{t-1}, r \in R_{t} .
\end{gathered}
$$

Aggregating terms involving $d_{k}$ and $r_{k}$ in (63), one obtains

$I_{0}^{R}-\sum_{i=1}^{t}\left(X_{i 0}^{R}+X_{i 0}^{D}\right)+r_{t}-\sum_{k=1}^{t-1} d_{k}\left[\sum_{i=k+1}^{t}\left(X_{i k}^{1 R}+X_{i k}^{1 D}\right)\right]-\sum_{k=1}^{t-1} r_{k}\left[-1+\sum_{i=k+1}^{t}\left(X_{i k}^{2 R}+\right.\right.$

$\left.\left.X_{i k}^{2 D}\right)\right] \geq 0 \quad 1 \leq t \leq T, d \in D_{t-1}, r \in R_{t}$,

which can be rewritten as

$I_{0}^{R}-\sum_{i=1}^{t}\left(X_{i 0}^{R}+X_{i 0}^{D}\right) \geq-r_{t}+\sum_{k=1}^{t-1} d_{k}\left[\sum_{i=k+1}^{t}\left(X_{i k}^{1 R}+X_{i k}^{1 D}\right)\right]+\sum_{k=1}^{t-1} r_{k}\left[-1+\sum_{i=k+1}^{t}\left(X_{i k}^{2 R}+\right.\right.$

$\left.\left.X_{i k}^{2 D}\right)\right] \quad 1 \leq t \leq T, d \in D_{t-1}, r \in R_{t}$.

Using the methodology described in Section 3, one can equivalently reformulate (64) as

$$
\begin{array}{ll}
I_{0}^{R}-\sum_{i=1}^{t}\left(X_{i 0}^{R}+X_{i 0}^{D}\right) \geq-\bar{r}_{t}+\sum_{k=1}^{t-1} \bar{d}_{k}\left[\sum_{i=k+1}^{t}\left(X_{i k}^{1 R}+X_{i k}^{1 D}\right)\right]+\sum_{k=1}^{t-1} \bar{r}_{k}\left[-1+\sum_{i=k+1}^{t}\left(X_{i k}^{2 R}+\right.\right. \\
\left.\left.X_{i k}^{2 D}\right)\right]+\sum_{k=1}^{t-1}\left(\hat{d}_{k} v_{t k}^{1}+\hat{r}_{k} v_{t k}^{2}\right)+\hat{r}_{t} & 1 \leq t \leq T \\
-v_{t k}^{1} \leq \sum_{i=k+1}^{t}\left(X_{i k}^{1 R}+X_{i k}^{1 D}\right) \leq v_{t k}^{1} & 1 \leq t \leq T, 1 \leq k \leq t-1 \\
-v_{t k}^{2} \leq 1-\sum_{i=k+1}^{t}\left(X_{i k}^{2 R}+X_{i k}^{2 D}\right) \leq v_{t k}^{2} & 1 \leq t \leq T, 1 \leq k \leq t-1 .
\end{array}
$$

Note that (65)-(67) are equivalent to (34)-(36). The term $\sum_{k=1}^{t-1}\left(\hat{d}_{k} v_{t k}^{1}+\hat{r}_{k} v_{t k}^{2}\right)+\hat{r}_{t}$ in (65) is due to the uncertainty in demand and returns. 
Production Planning with Remanufacturing under Uncertain Demand and Returns 297

(e) Constraints (37)-(39): Substituting the right-hand side of (18) in place of $X_{t}^{M}$ variable in $X_{t}^{M} \geq 0$ gives

$X_{t 0}^{M}+\sum_{k=1}^{t-1}\left(d_{k} X_{t k}^{1 M}+r_{k} X_{t k}^{2 M}\right) \geq 0 \quad 1 \leq t \leq T, d \in D_{t-1}, r \in R_{t-1}$.

Using the methodology described in Section 3, one can equivalently reformulate (68) as

$-X_{t 0}^{M}-\sum_{k=1}^{t-1}\left(\bar{d}_{k} X_{t k}^{1 M}+\bar{r}_{k} X_{t k}^{2 M}\right)+\sum_{k=1}^{t-1}\left(\hat{d}_{k} u_{t k}^{1 M}+\hat{r}_{k} u_{t k}^{2 M}\right) \leq 0 \quad 1 \leq t \leq T$,

$-u_{t k}^{1 M} \leq X_{t k}^{1 M} \leq u_{t k}^{1 M}$

$1 \leq t \leq T, 1 \leq k \leq t-1$

$-u_{t k}^{2 M} \leq X_{t k}^{2 M} \leq u_{t k}^{2 M}$

$1 \leq t \leq T, 1 \leq k \leq t-1$,

Note that (69) is equivalent to (37) when multiplied by -1 . Constraints (70) and (71) are the same as (38) and (39), respectively.

(f) Constraints (40)-(42) and (43)-(45): Similar to part (e). 